Messen, Kongresse

und Events

SPALTIMPR Ä GNIERUNG

\title{
Fertigung von CFK-Motorhauben \\ in wenigen Minuten
}

Wissenschaftler vom Institut für Kunststoffverarbeitung (IKV) und dem Institut für Kraftfahrzeuge (ika) der RWTH Aachen haben ein aus Kohlenstofffasern gefertigtes Musterbauteil einer Ford Focus-Motorhaube präsentiert. Im Vergleich zur serientypischen Stahlmotorhaube ist das Gewicht der CFK-Motorhaube um $60 \%$ auf unter $5 \mathrm{~kg}$ reduziert.

Die CFK-Motorhaube soll im Spaltimprägnierverfahren serientauglich produziert werden. Bereits 2009 wurde zusammen mit der Firma Hille Engineering eine entsprechende Anlage für die automatisierte Fertigung in kurzen Zykluszeiten von Hochleistungsbauteilen aus faserverstärkten Kunststoffen (FVK) entwickelt. Seitdem arbeitet das IKV kontinuierlich an der Ausweitung des Verfahrens. Aktuell können hochwertige Bauteile mit integrierten Funktionselementen im Maß von $50 \times 50 \mathrm{~cm}^{2}$ in weniger als fünf Minuten gefertigt werden. Für die CFK-Motorhaube des Ford Focus wurden die bisher gewonnenen Erkenntnisse auf die Dimension von $105 \times 158 \mathrm{~cm}^{2}$ übertragen. Für die Fertigung werden unter 15 Minuten veranschlagt.

Das Bauteil umfasst neben der gekrümmten Bauteilkontur und Versteifungsstrukturen auch Anbindungselemente für die Montage der Motorhaube. Bei der Auslegung spielten die mechanischen Eigenschaften, wie der Fußgängerschutz, die Materialauswahl, der Temperatureinsatzbereich und die Bauteiloberflächenqualität, eine entscheidende Rolle. Nach der Ermittlung der mechanischen Kennwerte aus dem vorher ausgewählten Harz- und Halbzeugsystem wurde die
Motorhaube bezüglich Steifigkeits- und Crasheigenschaften am ika simulativ ausgelegt. Die tatsächlichen Eigenschaften wurden anhand von Steifigkeits- und Crashprüfungen ermittelt. Die Testergebnisse bestätigen die Endgeometrie des Bauteils.

Auf Basis dieser Ergebnisse wird ein spezielles Werkzeug für die im IKV-Technikum installierte Spaltimprägnieranlage konstruiert. Nach der Inbetriebnahme des Werkzeugs wird am IKV weiter an Prozessoptimierungen bezüglich Prozessgeschwindigkeit und Bauteiloberflächenqualität geforscht. Es werden Prototypen mit der auf die Motorhaubenfertigung umgerüsteten Spaltimprägnieranlage gefertigt. Diese werden dann speziellen Tests, beispielsweise zum Kopfaufprallschutz oder zur Temperaturbelastung, unterzogen, die für eine Serienzulassung erforderlich sind.

An dem Gemeinschaftsprojekt sind neben IKV und ika die Industriepartner Ford Forschungszentrum Aachen, Composite Impulse, Toho Tenax Europe, Evonik Industries und Henkel beteiligt. Es wird vom Land Nordrhein-Westfalen gefördert. Den Abschluss soll das Projekt mit der seriennahen und zykluszeitoptimierten Fertigung der Motorhaube im Spaltimprägnierverfahren zum September 2013 finden.

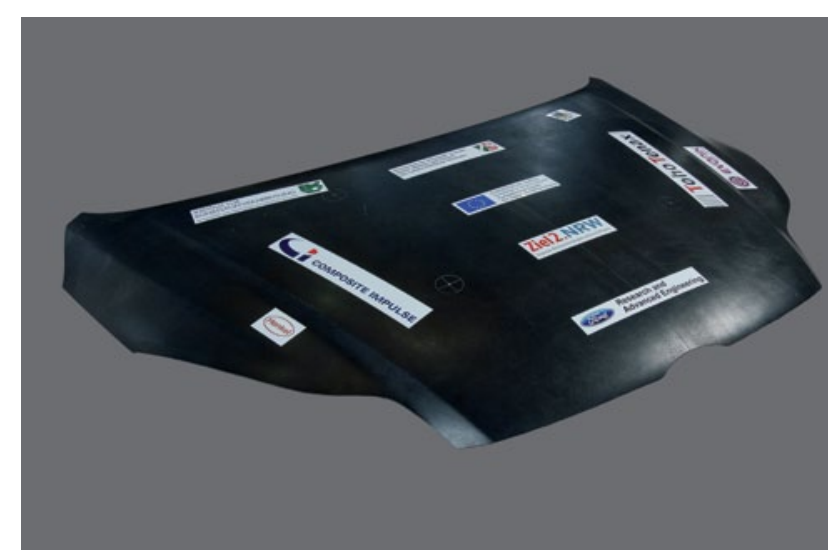

lieben Salzburg.
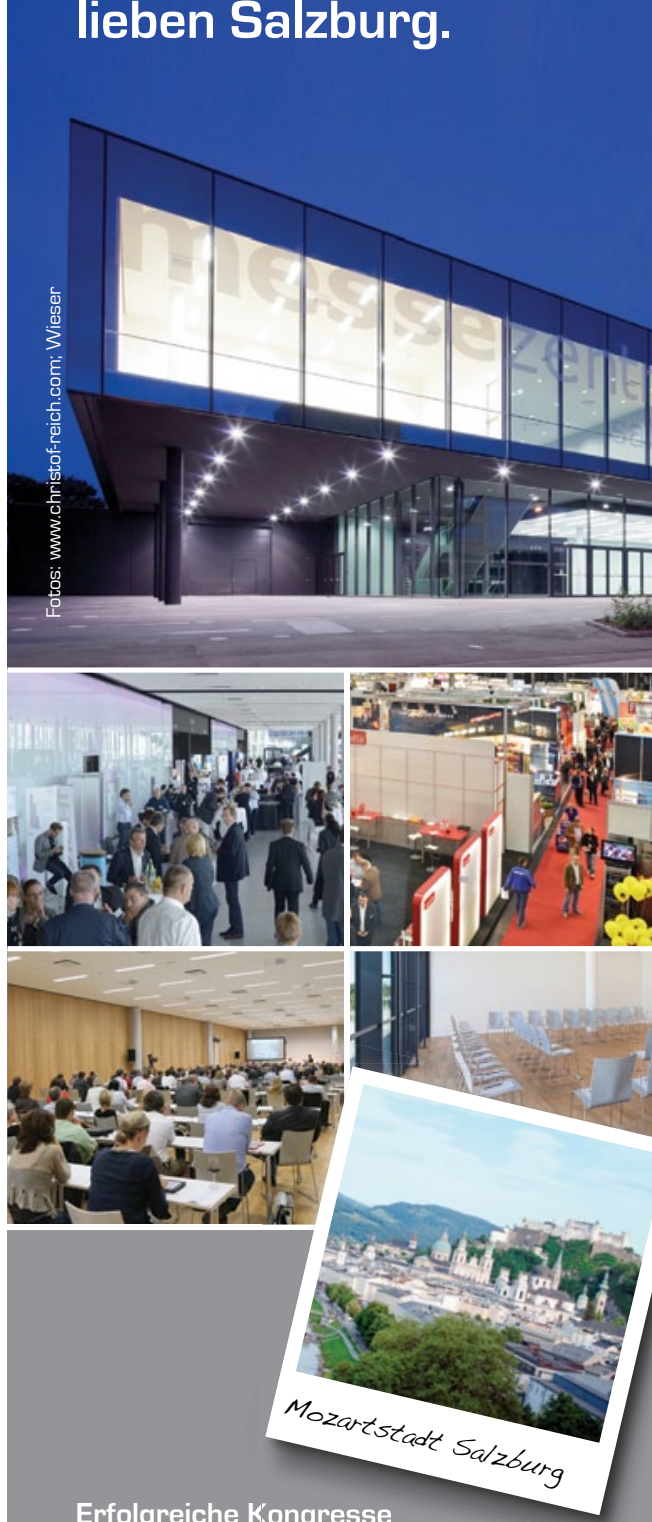

Erfolgreiche Kongresse

im neuen Tagungsbereich des

Messezentrum Salzburg!

Die neue Multifunktionshalle verbunden mit der Salzburgarena und den weiteren Messehallen bietet Ihnen ideale Kombinationsmöglichkeiten für Ihre Veranstaltung.

Lassen Sie sich begeistern von moderner Infrastruktur, persönlichem Service und einem professionellen Networking wir bieten Ihnen eine Plattform für Ihre erfolgreichen Kontakte!

\section{Messezentrum Salzburg GmbH T: +43 66224040 \\ office@messezentrum-salzburg.at www.messezentrum-salzburg.at}

messen · kongresse · events 